Article

\title{
Surface Modification of Hemoglobin-Based Oxygen Carriers Reduces Recognition by Haptoglobin, Immunoglobulin, and Hemoglobin Antibodies
}

\author{
Ausanai Prapan ${ }^{1,2}$, Nittiya Suwannasom ${ }^{1,3}$, Chiraphat Kloypan ${ }^{1,4}(\mathbb{D}$, \\ Saranya Chaiwaree 1,5 (D), Axel Steffen ${ }^{1}$, Yu Xiong ${ }^{1}$, Ijad Kao ${ }^{1}$, Axel Pruß ${ }^{1}$, \\ Radostina Georgieva 1,6 ${ }^{(D)}$ and Hans Bäumler 1,*(D) \\ 1 Institute of Transfusion Medicine, Charité-Universitätsmedizin Berlin, 10117 Berlin, Germany \\ 2 Department of Radiological Technology, Faculty of Allied Health Sciences, Naresuan University, \\ Phitsanulok 65000, Thailand \\ 3 Division of Biochemistry and Nutrition, School of Medical Sciences, University of Phayao, \\ Phayao 56000, Thailand \\ 4 Division of Clinical Immunology and Transfusion Sciences, School of Allied Health Sciences, \\ University of Phayao, Phayao 56000, Thailand \\ 5 Department of Pharmaceutical Technology, Faculty of Pharmacy, Payap University, \\ Chiang Mai 50000, Thailand \\ 6 Department of Medical Physics, Biophysics and Radiology, Medical Faculty, Trakia University, \\ Stara Zagora 6000, Bulgaria \\ * Correspondence: hans.baeumler@charite.de; Tel.: +49-30-450-525-131
}

Received: 3 July 2019; Accepted: 17 July 2019; Published: 21 July 2019

\begin{abstract}
Hemoglobin-based oxygen carriers (HBOCs) represent a propitious type of blood substitute to transport oxygen throughout the body while acting as a carrier in biomedical applications. However, HBOCs in blood are recognized and rapidly scavenged by the body's innate immune systems. To overcome this problem, HBOCs require a surface modification that provides protection against detection and elimination in order to prolong their circulation time after administration. In this study, we investigated different surface modifications of hemoglobin submicron particles (HbMPs) by double/triple precipitation, as well as by adsorption of human serum albumin (HSA), hyaluronic acid (HA), and pluronic (Plu) to discover how diverse surface modifications influence the oxygen binding capacity and the binding of anti-hemoglobin $(\mathrm{Hb})$ antibodies, immunoglobulin $\mathrm{G}$ (IgG), and haptoglobin (HP) to HbMPs. The particle size and zeta potential of the six types of $\mathrm{HbMP}$ modifications were analyzed by zeta sizer, confocal laser scanning microscopy, and transmission electron microscopy (TEM), and were compared to the unmodified HbMPs. The results revealed that all surface-modified $\mathrm{HbMPs}$ had a submicron size with a negative charge. A slight decrease in the oxygen binding capacity was noticed. The specific binding of anti-Hb antibodies, IgG, and $\mathrm{HP}$ to all surface-modified HbMPs was reduced. This indicates a coating design able to protect the particles from detection and elimination processes by the immune system, and should lead to a delayed clearance and the required and essential increase in half-life in circulation of these particles in order to fulfill their purpose. Our surface modification method reflects a promising strategy for submicron particle design, and can lead the way toward novel biomedical applications.
\end{abstract}

Keywords: hemoglobin; blood substitute; haptoglobin; serum albumin; immunoglobulin G; hyaluronic acid

\section{Introduction}

Blood transfusion is a potentially life-saving procedure and is used in many therapeutic applications. Several risks, such as transmission of infectious disease, immunomodulation etc., have 
accelerated the development of blood substitutes as alternatives to human blood [1,2]. The development of blood substitutes has mainly focused on the requirements that they carry oxygen, have no toxicity or side effects, and have a sufficient circulation time [3-5]. During recent decades, the use of blood substitutes has been extensively investigated especially with the attention to hemoglobin-based oxygen carriers (HBOCs). Interestingly, the development and modification of HBOCs for clinical trials is still in progress [5-7]. Currently, the different approaches to fabricating HBOCs that have been investigated have had the primary consideration of stabilizing the hemoglobin $(\mathrm{Hb})$ molecule and improving the oxygen-carrying capabilities by cross-linked hemoglobin, polymerized hemoglobin, hemoglobin conjugated to macromolecules, and encapsulated hemoglobin $[8,9]$. HBOCs are expected to increase the total oxygen carrying capacity and oxygen delivery of blood because of an increase in total $\mathrm{Hb}$ concentration. Therefore, the oxygen carrying behavior of HBOCs should be considered and investigated.

However, one main problem seems to be the short half-life of HBOCs after their transfusion [10]. Surprisingly, there are no literature data available, which could explain the reason for the elimination of the HBOCs. It is known that $\mathrm{Hb}$ is detected by haptoglobin (HP), and the $\mathrm{Hb}-\mathrm{HP}$ complex is recognized by Kupffer cells very fast [11,12]. Therefore, HBOCs should preclude $\mathrm{Hb}-\mathrm{HP}$ complex formation to avoid liver uptake and depletion of HP in the plasma. Additionally, we have to consider the opsonization of HBOCs, which would result in a rapid clearance by the reticuloendothelial system (RES). To overcome this obstacle, modifications of the surface properties of carriers, also known as "stealth coatings," have been developed and applied to prevent particles from protein absorption and immune recognition $[13,14]$. The properties of these polymers should include high flexibility and high hydrophilicity. Many materials and coating approaches have been utilized for modifying the surface of particles. For instance, the natural polysaccharides (e.g., hyaluronic acid) or synthetic polymers (e.g., polyethylene glycol (PEG)-based copolymers such as pluronic) have been widely used as coating materials. Hyaluronic acid (HA) or hyaluronan is a hydrophilic linear glycosaminoglycan composed of alternating $(1 \rightarrow 4)-\beta-\mathrm{d}$-glucuronic acid and $(1 \rightarrow 3)-\beta-\mathrm{N}$-acetyl-d-glucosamine [15], which has been used for the fabrication of hyaluronic-acid-based layer-by-layer capsules for the intracellular uptake of the capsules by macrophages, indicating their potential as a carrier for intracellular drug delivery [16]. Pluronics (poloxamers) are a class of water-soluble and non-ionic A-B-A triblock copolymers, where A is poly (ethylene oxide) (PEO) and B is poly (propylene oxide) (PPO). Pluronic block copolymers have been used and applied as a micellar nano-carrier for drug delivery system, resulting in increased solubility and metabolic stability, and improving circulation time. Gyulai et al. reported that the presence of pluronics on the surface of poly lactic-co-glycolic acid (PLGA) nanoparticles preserved the aggregation stability and improved the nonspecific membrane affinity of the system [17].

Amongst the available coating materials, human serum albumin (HSA) is also a promising molecule for surface modification. HSA is the product of hepatic protein synthesis and is the most abundant plasma protein (molecular weight $(M W)=66.5 \mathrm{kDa}$ ). These properties, including high stability, preferential uptake in tumor and inflamed tissue, biodegradability, and lack of toxicity and immunogenicity indicate a good material for drug delivery carriers [18,19]. Esfandyari-Manes et al. also reported that the surface modification of particles with albumin improves long-circulating and stealth particles for drugs delivery [20].

To date, there has been a great interest in the development of HBOCs in their versatile properties, including biocompatibility, biodegradability, and low immunogenicity. Previously published data of hemoglobin particles synthesized by a co-precipitation-crosslinking-dissolution technique (CCD-technique) demonstrated HBOCs with high oxygen affinity and low immunogenicity [21,22].

The aim of this study was to clarify in which way several modifications of the surface of $\mathrm{HbMP}$ influence the oxygen binding and the adsorption of antibodies, especially the anti- $\mathrm{Hb}$ antibodies, immunoglobulin, and the glycoprotein HP. The surface modifications were performed by double/triple precipitation, as well as by adsorption of human serum albumin (HSA), hyaluronic acid (HA), and pluronic (Plu). As mentioned above, the utilization of HSA, HA, and Plu as surface coating materials 
is a promising approach for drug delivery carriers. We hypothesized that these polymers could be applied to the particle and HbMP would become coated with a layer that would allow it to avoid anti-Hb antibody, IgG, and $\mathrm{HP}$ binding.

\section{Materials and Methods}

\subsection{Materials}

HSA was purchased from the Baxalta Deutschland $\mathrm{GmbH}$, Unterschleissheim, Germany. Manganese chloride tetrahydrate $\left(\mathrm{MnCl}_{2} \cdot 4 \mathrm{H}_{2} \mathrm{O}\right)$, sodium carbonate $\left(\mathrm{Na}_{2} \mathrm{CO}_{3}\right)$, ethylene-diaminetetraacetic acid (EDTA), sodium borohydride $\left(\mathrm{NaBH}_{4}\right)$, glutaraldehyde (GA), hyaluronic acid sodium salt, kolliphor ${ }^{\circledR} \mathrm{P} 407$, and potassium ferricyanide $\left(\mathrm{K}_{3}\left(\mathrm{Fe}(\mathrm{CN})_{6}\right)\right)$ were purchased from Sigma-Aldrich, Munich, Germany. Sodium chloride ( $\mathrm{NaCl}$ ) was purchased from Merck KGaA, Darmstadt, Germany. Ringer's acetate solution (consisting of sodium chloride, potassium chloride, calcium chloride dehydrate, magnesium chloride hexahydrate, and sodium acetate trihydrate in water) was purchased from Serumwerk Bernburg AG, Bernburg, Germany. Ampuwa ${ }^{\circledR}$ (aqua ad injectable) was purchased from Fresenius Kabi Deutschland GmbH, Bad Homburg, Germany. Pronase was purchased from Roche Diagnostics GmbH, Mannheim, Germany. Rabbit anti-bovine hemoglobin antibody (LS-C294442) and allophycocyanin (APC)-conjugated rabbit polyclonal antibody to human haptoglobin (LS-C236382) were purchased from LS Bio, Washington, WA, USA. Goat anti-rabbit IgG conjugated to SureLight ${ }^{\circledR}$ APC (D3-114-1mg) were purchased from Columbia Biosciences, Frederick, MD, USA. Haptoglobin from pooled human plasma (H3536) was purchased from Sigma, Saint Louis, MO, USA. Human IgG isotype control (biotin), (NBP1-96855) was purchased from Novus Biologicals a Bio-Techne Brand, Centennial, CO, USA. APC-streptavidin (Cat\#405243, Lot B223923) was purchased from BioLegend Inc., San Diego, CA, USA.

Hemoglobin $(\mathrm{Hb})$ was extracted from bovine red blood cells by hypotonic hemolysis [23]. Fresh bovine whole blood (received from Biophyll $\mathrm{GmbH}$, Dietersburg, Germany), anticoagulated with EDTA, was centrifuged $\left(2500 \mathrm{~g}, 10 \mathrm{~min}, 4^{\circ} \mathrm{C}\right)$, was washed three times with phosphate-buffered saline (PBS), and then five volumes of ice-cold Ampuwa were added to one volume of washed red blood cells. The solution was stirred at $4{ }^{\circ} \mathrm{C}$ overnight and then centrifuged $\left(10,000 \mathrm{~g}, 4{ }^{\circ} \mathrm{C}\right)$ for $1 \mathrm{~h}$. The supernatant was filtered through a $0.1 \mu \mathrm{m}$ polyethersulfone filter (Sartorius AG, Göttingen, Germany) and stored at $-80{ }^{\circ} \mathrm{C}$ as a stock solution for further use.

\subsection{Particle Preparation}

The synthesis and surface modification of HbMPs are schematically displayed in Figure 1. We fabricated six different types of surface-modified HbMP. A short description of each particle type and the corresponding abbreviations are listed in Table 1.

Table 1. Specification of the different surface modifications of HbMPs.

\begin{tabular}{lc}
\hline \multicolumn{1}{c}{ Particle Type } & Abbreviation \\
\hline Hemoglobin submicron particles & HbMPs \\
Double precipitated HbMP in presence of HSA: & HSA-d-HbMP \\
Hb precipitation first, HSA absorption before second precipitation step. & HA-d-HbMP \\
$\begin{array}{l}\text { Double precipitated HbMP in presence of HA: } \\
\text { Hb precipitation first, HA and HSA absorption before second precipitation step. }\end{array}$ & Plu-d-HbMP \\
$\begin{array}{l}\text { Double precipitated HbMP in presence of pluronic: } \\
\text { Hb precipitation first, Plu and HSA absorption before second precipitation step. }\end{array}$ & HSA-t-HbMP \\
Triple precipitated HbMP in presence of HSA: & \\
Hb precipitation first, HSA absorption before second and third precipitation step. & HA-t-HbMP \\
Triple precipitated HbMP in presence of HA: & \\
Triple precipitation first, HA and HSA absorption before second and third precipitation step. & Plu-t-HbMP \\
Hb precipitation first, Plu and HSA absorption before second and third precipitation step. & \\
\hline
\end{tabular}




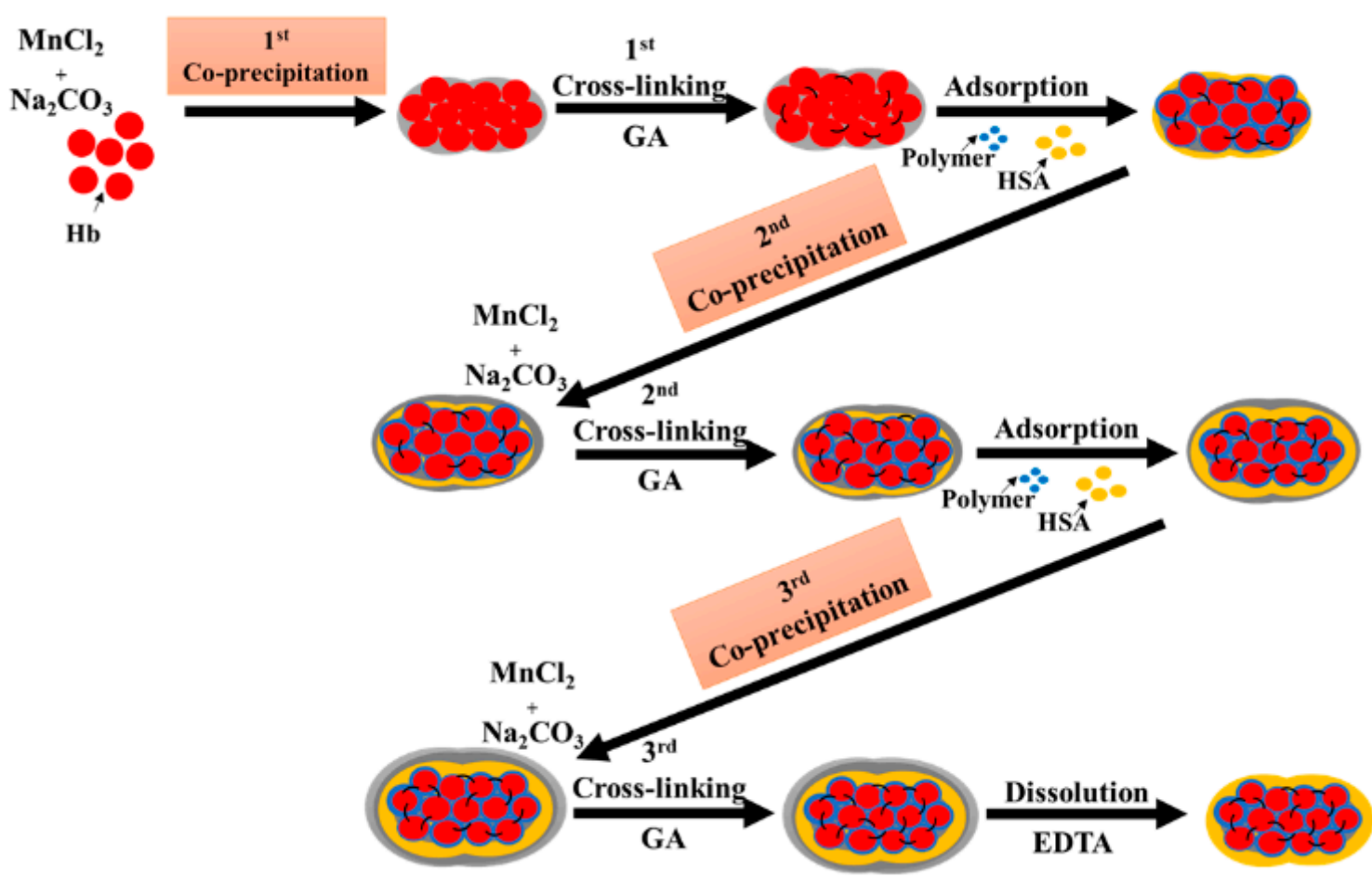

Figure 1. Fabrication scheme of surface-modified hemoglobin submicron particles (HbMPs) by triple precipitation in presence of polymers (human serum albumin (HSA), hyaluronic acid (HA), or pluronic (Plu)). For fabrication of the double precipitated surface-modified HbMP, the particles were dissolved with ethylene-diaminetetraacetic acid (EDTA) after the second crosslinking step.

\subsubsection{Preparation of Hemoglobin Submicron Particles (HbMPs)}

HbMPs were fabricated using a protocol based on the CCD technique, as previously described [24,25]. Briefly, $5 \mathrm{mg} / \mathrm{mL} \mathrm{Hb}$ in $0.125 \mathrm{M} \mathrm{MnCl}_{2}$ solution was mixed rapidly with $0.125 \mathrm{M}$ $\mathrm{Na}_{2} \mathrm{CO}_{3}$ and stirred for $30 \mathrm{~s}$ to obtain $\mathrm{Hb}-\mathrm{MnCO}_{3}$ particles (first precipitation). These particles were washed twice with distilled water by centrifugation $(3000 \mathrm{~g}, 3 \mathrm{~min}$ ) and re-dispersed in $0.9 \% \mathrm{NaCl}$ solution. The $\mathrm{Hb}-\mathrm{MnCO}_{3}$ particles were incubated with $0.02 \% \mathrm{GA}$ solution for $1 \mathrm{~h}$ at room temperature. After that, the obtained particles were washed and re-suspended in distilled water. These particles were used for a second as well as a third precipitation.

\subsubsection{Preparation of HSA-d-HbMPs and HSA-t-HbMPs}

Double precipitated HbMPs (HSA-d-HbMP): For the second co-precipitation step, the washed $\mathrm{Hb}-\mathrm{MnCO}_{3}$ particles were suspended in $0.04 \mathrm{M} \mathrm{MnCl}_{2}$ and $0.4 \% \mathrm{HSA}$ was added to adsorb onto the surface of the particles for $5 \mathrm{~min}$. After adding $0.04 \mathrm{M} \mathrm{Na}_{2} \mathrm{CO}_{3}$, the obtained particles were washed with distilled water by centrifugation $(3000 \mathrm{~g}, 3 \mathrm{~min}$ ) and were re-suspended in $0.9 \% \mathrm{NaCl}$ solution. The double co-precipitated $\mathrm{HSA}-\mathrm{d}-\mathrm{Hb}-\mathrm{MnCO}_{3}$ particles were cross-linked again with $0.02 \% \mathrm{GA}$ at room temperature under moderate stirring. A measure of $0.25 \mathrm{M}$ EDTA was used to dissolve the $\mathrm{MnCO}_{3}$ templates. A measure of $0.2 \mathrm{mg} / \mathrm{mL} \mathrm{NaBH}_{4}$ in $0.1 \mathrm{M} \mathrm{NaOH}$ solution was added and incubated for $30 \mathrm{~min}$. The resulting HSA-d-HbMPs were washed three times with $0.9 \% \mathrm{NaCl}$ solution supplemented with $0.2 \%$ HSA (centrifugation 10,000 g, $10 \mathrm{~min}$ ), and were re-suspended in Ringer's acetate solution and stored at $4{ }^{\circ} \mathrm{C}$ for further use.

Triple precipitated $\mathrm{HbMPs}$ (HSA-t-HbMP): The additional co-precipitation step of $\mathrm{MnCO}_{3}$ with HSA for fabrication of HSA-t-HbMPs was performed after the second crosslinking step, followed by cross-linking with GA in the same manner and concentrations before performing the dissolution step. 


\subsubsection{Preparation of HA-d-HbMPs and HA-t-HbMPs}

The HA-d-HbMPs were prepared in the same manner as HSA-d-HbMPs, using $0.04 \%$ HA in addition to the $0.4 \% \mathrm{HSA}$. The fabrication of HA-t-HbMPs was performed after the second crosslinking step by co-precipitation of $\mathrm{MnCO}_{3}$ with $0.04 \% \mathrm{HA}$ and $0.4 \%$ has, followed by cross-linking with GA in the same manner and concentrations before performing the dissolution step.

\subsubsection{Preparation of Plu-d-HbMPs and Plu-t-HbMPs}

The Plu-d-HbMPs were also prepared in the same manner as HSA-d-HbMPs, using $0.4 \%$ pluronic in addition to the $0.4 \%$ HSA. The fabrication of Plu-t-HbMPs was performed after the second crosslinking step by co-precipitation of $\mathrm{MnCO}_{3}$ with $0.4 \%$ pluronic and $0.4 \%$ has, followed by cross-linking with GA in the same manner and concentrations before performing the dissolution step.

\subsection{Particle Characterization}

\subsubsection{Size and Zeta-Potential}

The hydrodynamic size and zeta potential of each surface-modified HbMPs was determined using a Zetasizer Nano ZS instrument (Malvern Instruments Ltd., Worcestershire, UK). Each sample was diluted 1:200 using PBS solutions. All the measurements were carried out in triplicate.

\subsubsection{Transmission Electron Microscopy (TEM)}

TEM imaging was performed using a Zeiss EM 906 Omega microscope (Carl Zeiss AG, Oberkochen, Germany) at an acceleration voltage of $80 \mathrm{kV}$. Sample preparation was as follows: The water of the particle suspensions was exchanged with ethanol by washing steps with gradually increasing ethanol concentrations $(10 \%, 20 \%, 40 \%, 60 \%, 80 \%, 99.9 \%)$. After the last centrifugation (1800 g, $6 \mathrm{~min})$, the sample was infiltrated with LR-White Resin (ratio of Ethanol: LR-WR =1:1) and incubated overnight at room temperature. After that, the sample was exchanged with fresh LR-WR and was transferred into gelatin capsules. Polymerization of the resin was performed with UV light for $48 \mathrm{~h}$ at room temperature. At the end of the process, ultrathin sections ( $60-70 \mathrm{~nm}$ on mesh-grids) were prepared. Sections were stained with $1 \%$ phosphotungstic acid for $15 \mathrm{~min}$ and $4 \%$ aqueous uranyl acetate for $15 \mathrm{~min}$.

\subsubsection{Confocal Laser Scanning Microscopy (CLSM)}

CLSM images of each surface-modified HbMPs preparation were taken with LSM 510 Meta (Carl Zeiss MicroImaging GmbH, Jena, Germany) microscope with a 100× oil-immersion objective (numerical aperture 1.3), applying excitation wavelength of $488 \mathrm{~nm}$ and a long pass emission filter of $505 \mathrm{~nm}$.

\subsubsection{Determination of $\mathrm{Hb}$ Content}

The total hemoglobin concentration of the surface-modified HbMPs was determined by the modified alkaline hematin-D method (AHD method) [26,27]. Briefly, $2 \%(v / v)$ particle suspensions were mixed with pronase to obtain a final concentration of $0.5 \mathrm{mg} / \mathrm{mL}$ pronase, and were incubated at $45^{\circ} \mathrm{C}$ for $30 \mathrm{~min}$. After that, AHD reagent $(25 \mathrm{mg} / \mathrm{mL}$ Triton X-100 in $0.1 \mathrm{M} \mathrm{NaOH})$ was added at the volume ratio 1:1, then thoroughly mixed and incubated for $15 \mathrm{~min}$ at room temperature. All samples were measured at the absorption spectra at wavelength $574 \mathrm{~nm}$ using a UV-vis spectrophotometer (Hitachi U2800, Hitachi High-Technologies Corporation, Tokyo, Japan), and an average of three individual measurements were recorded for calculation of the $\mathrm{Hb}$ concentration. 


\subsubsection{Determination of Oxygenated HbMPs (Oxy-Hbs)}

The ferricyanide method was applied for measurement of the oxygen content on the surface-modified HbMPs $[28,29]$. Briefly, $2 \%(v / v)$ particle suspensions was placed into a glass vial containing a magnetic stirrer. A miniaturized optical needle type oxygen sensor (oxygen micro-sensor NTH-PSt7, PreSens-Precision Sensing GmbH, Regensburg, Germany) connected to a portable oxygen meter with data logging (Microx 4, PreSens-Precision Sensing GmbH, Regensburg, Germany) was inserted into the stirred sample, and the concentration of dissolved oxygen was recorded. After $3 \mathrm{~s}$, $10 \%$ potassium ferricyanide was added and the change of the concentration of dissolved oxygen was measured until a stable value was reached. The difference between the final $p \mathrm{O}_{2}$ value and the initial $p \mathrm{O}_{2}$ corresponds to the oxygen bound to hemoglobin in the particles. The change in $p \mathrm{O}_{2}$ was converted into the concentration of released oxygen $\left(\mathrm{cO}_{2}\right)$ with the following formula, known as Henry's Law:

$$
c \mathrm{O}_{2}=a \mathrm{O}_{2} \times p \mathrm{O}_{2}
$$

where $a \mathrm{O}_{2}$ is the solubility coefficient of oxygen in blood: $0.0031 \mathrm{~mL} \mathrm{O}_{2} / \mathrm{mmHg} \mathrm{O}_{2} / \mathrm{dL}$ blood. Having derived the mass of released oxygen, the concentration of the $\mathrm{Oxy}-\mathrm{Hb}$ was calculated as follows:

$$
c(\mathrm{Oxy}-\mathrm{Hb})=\frac{m \mathrm{O}_{2}}{\rho\left(\mathrm{O}_{2}\right) \times H b_{s}}
$$

where $\rho \mathrm{O}_{2}=1.43 \mathrm{~g} / \mathrm{L}$ is the density of $\mathrm{O}_{2}$ and $\mathrm{Hb}_{\mathrm{s}}$ is the saturated $\mathrm{Oxy}-\mathrm{Hb}$ content which equals 1.34 $\mathrm{mL}$ of $\mathrm{O}_{2}$ per gram of $\mathrm{Hb}$.

\subsection{In Vitro Antibody Binding Assays}

To confirm a successful surface modification of $\mathrm{HbMP}$ and detect the $\mathrm{Hb}$ on the surface of $\mathrm{HbMP}$, the adsorption of anti-bovine $\mathrm{Hb}$ antibodies, immunoglobulins (IgG), and haptoglobin (HP) was performed by using flow cytometry (FACS Canto II, Becton \& Dickinson, Franklin Lakes, NJ, USA). In this experiment, we used the six different types of surface modified HbMPs and the unmodified HbMPs as a control (description is shown in Table 1). Staining the particles with only secondary antibodies provided the non-specific binding, staining with both primary and secondary antibodies provided the total binding. Specific binding was calculated by subtracting the non-specific binding from the total binding.

\subsubsection{Binding Assay of Anti-Hb Antibodies}

Samples of $1 \mathrm{~mL}$ of $0.2 \%(v / v)$ particle suspensions were incubated for $2 \mathrm{~h}$ with the primary rabbit anti-bovine hemoglobin antibody at a final concentration of $2.5 \mu \mathrm{g} / \mathrm{mL}$. The particle suspensions were then incubated with the secondary APC-conjugated goat anti-rabbit IgG (final concentration $1 \mu \mathrm{g} / \mathrm{mL}$ ). Incubations were performed at room temperature. Between each incubation step, the particle suspensions were washed 2-3 times with PBS solution. Subsequently, the particle suspensions were incubated again in darkness for $2 \mathrm{~h}$, washed, and re-suspended in $300 \mu \mathrm{L}$ PBS solutions for investigation by flow cytometry.

\subsubsection{Binding Assay of Immunoglobulin (IgG)}

The binding of IgG to particles was also performed, and comprised the same step procedure described above used for the binding assay of anti- $\mathrm{Hb}$ antibodies. The primary biotin conjugated human IgG isotype control was added into $1 \mathrm{~mL}$ of particle suspension at a final concentration of $2.5 \mu \mathrm{g} / \mathrm{mL}$, and was incubated for $2 \mathrm{~h}$ at room temperature followed by PBS washings. After that, the particle suspensions were incubated for $2 \mathrm{~h}$ in the dark with APC-streptavidin at a final concentration of $1 \mu \mathrm{g} / \mathrm{mL}$. 


\subsubsection{Binding Assay of Haptoglobin (HP)}

The accessibility of $\mathrm{Hb}$ of each surface-modified HbMPs for the HP was investigated in the same manner as described above. Firstly, HP from pooled human plasma was added to $1 \mathrm{~mL}$ of particle suspension at a final concentration of $2.5 \mu \mathrm{g} / \mathrm{mL}$ HP. Next, APC-conjugated rabbit polyclonal antibody to human haptoglobin (HP antibody) was added at a final concentration of $1 \mu \mathrm{g} / \mathrm{mL}$ (secondary antibody).

\subsection{Statistical Analysis}

The results are presented as means \pm standard deviation (SD). The data were compared using ANOVA-like test. GraphPad Prism 6 software (GraphPad, La Jolla, CA, USA) was employed for graphs and statistical analyses. $p$-value $<0.05$ were considered statistically significant.

\section{Results and Discussion}

\subsection{Particle Characterization}

\subsubsection{Particle Morphology and Size}

The morphology of HbMPs and surface-modified HSA-d-HbMPs and HSA-t-HbMPs was investigated by TEM. The HbMPs, HSA-d-HbMPs, and HSA-t-HbMPs showed a peanut-like shape (Figure 2A-C, respectively). The unmodified particles (Figure 2A) clearly showed a more porous structure compared to the surface-modified HbMPs with HSA. The HSA-d-HbMPs and HSA-t-HbMPs appeared denser and were surrounded by a thin dark rim, which confirms the successful coating with HSA. Due to the autofluorescence caused by the protein crosslinking with GA, the HbMPs, HSA-d-HbMPs and HSA-t-HbMPs could be additionally visualized by CLSM. The corresponding CLSM images (Figure 2 inserts) confirm the suspension stability of the samples, e.g., absence of aggregation. This was also found for HbMPs with HA and pluronic surface modification, respectively (Supplementary Figure S1).

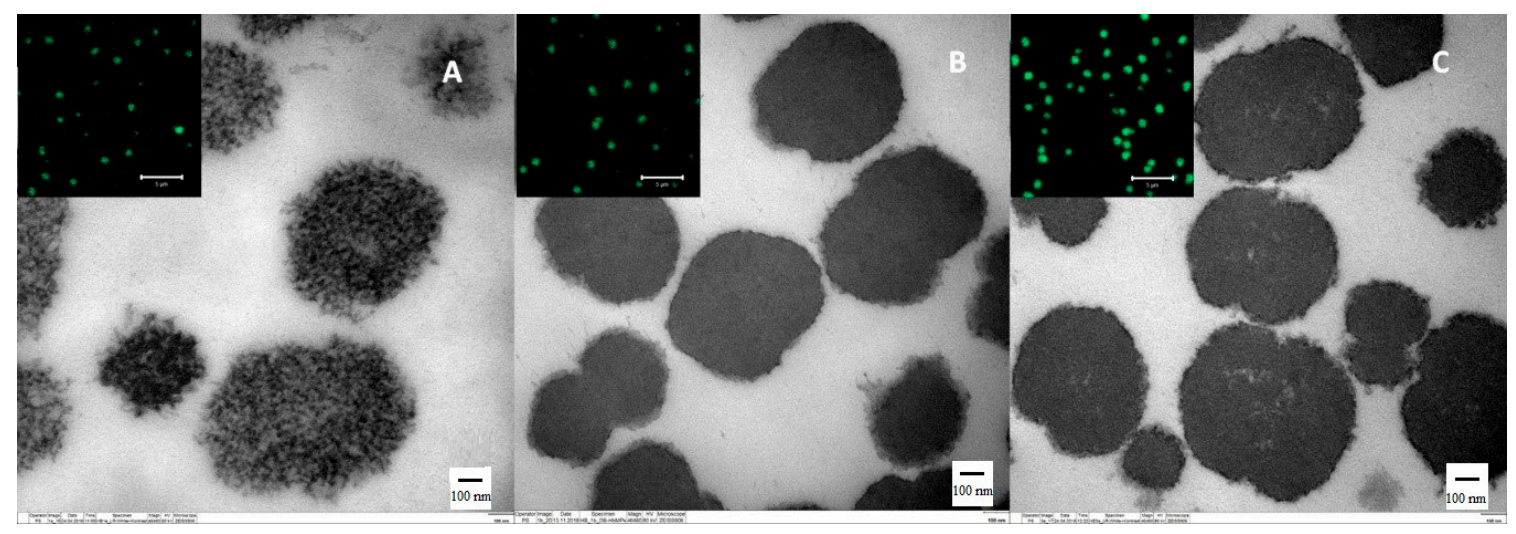

Figure 2. Transmission electron microscope (TEM) images (scale bar: $100 \mathrm{~nm}$ ) together with confocal laser scanning microscope (CLSM) imaging (inserts, scale bar: $5 \mu \mathrm{m}$ ) of unmodified hemoglobin submicron particles (HbMPs) (A), double precipitated HbMPs (HSA-d-HbMPs) (B), and triple precipitated HbMPs (HSA-t-HbMPs) (C).

The hydrodynamic diameter of all seven samples was determined by dynamic light scattering, and was in the range of 0.7 to $1 \mu \mathrm{m}$ with a low polydispersity index (PDI $<0.3$ ), indicating a narrow size distribution and an absence of aggregation. The hydrodynamic diameters of the surface-modified particles did not significantly differ from that of HbMPs (Figure 3A). 


\subsubsection{Zeta Potential}

The mean values of the zeta-potential of all surface-modified HbMPs suspended in PBS solutions (conductivity $18-20 \mathrm{mS} / \mathrm{cm}$ ) were negative (in the range -11 to $-13 \mathrm{mV}$ for HSA-d-HbMPs, HSA-t-HbMPs, Plu-d-HbMPs, and Plu-t-HbMPs). No significant differences in the zeta potential of the surface-modified HbMPs in presence of HSA and Plu could be found compared to $\operatorname{HbMPs}(p<0.05)$. However, the zeta potential of HA-d-HbMPs and HA-t-HbMPs became significantly more negative (increased by 36\%-38\%) compared to $\mathrm{HbMP}$ (Figure 3B). Similar results are reported in our previous studies about the influence of $\mathrm{HA}$ on $\mathrm{MnCO}_{3}$-templated $\mathrm{Hb} / \mathrm{BSA}-\mathrm{HA}$ particles [30]. The enhanced zeta potential values of the HA-d-HbMPs and HA-t-HbMPs confirm the attachment of HA, a strongly negatively charged polysaccharide, on the surface of the particles.
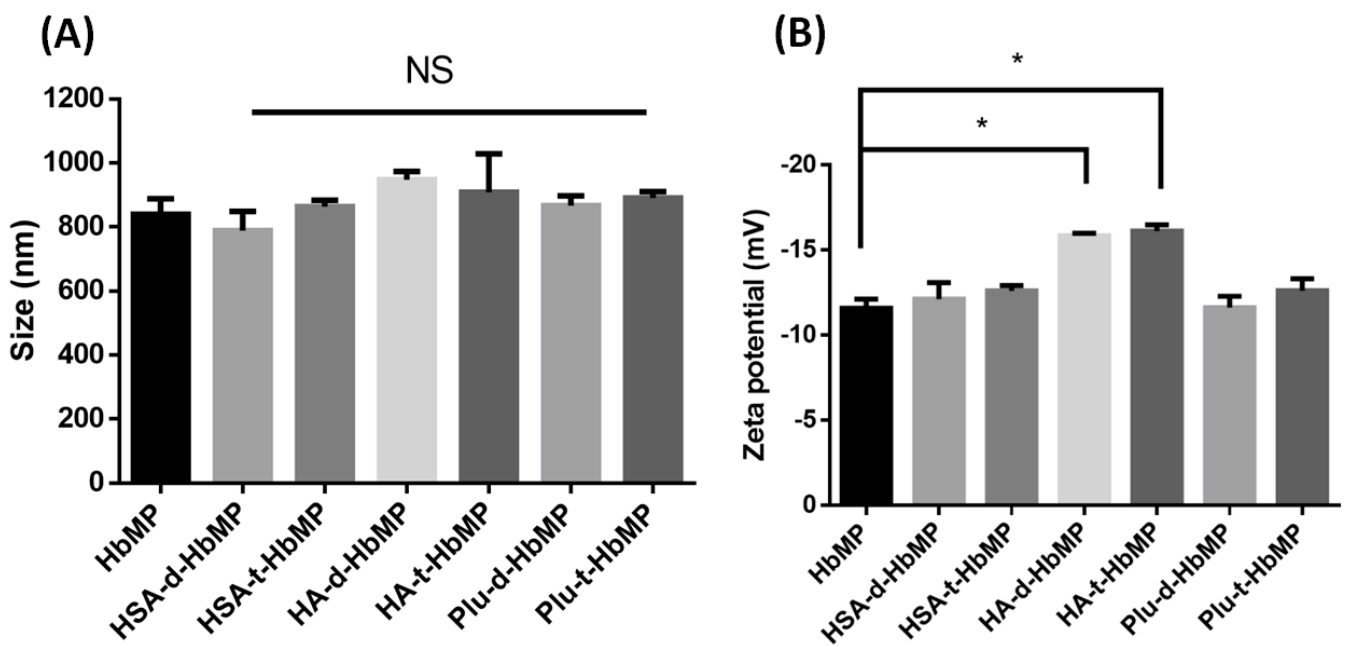

Figure 3. The hydrodynamic diameter (A) and zeta-potential (B) of the surface-modified HbMPs. Data are presented as mean $\pm \mathrm{SD}(n=3)$. "NS" indicates not significant, whereas the asterisk indicates a significant difference when compared to HbMPs $\left({ }^{*} p<0.05\right)$.

\subsubsection{Hemoglobin Content and Oxygen Binding Capacity}

The total $\mathrm{Hb}$ concentration of all surface-modified $\mathrm{HbMPs}$ revealed no statistically significant difference compared to HbMPs (35-40 mg/mL as calculated for suspensions with a hematocrit (Hct) of $20 \%, p$ value $<0.05$ ) (Figure $4 \mathrm{~A}$ ).

The oxygen binding capacity of HBOC is commonly determined by oxygen dissociation curves (ODC), which demonstrate the relationship between oxygen partial pressure and the oxygen fractional saturation [8]. However, this method requires specific and expensive equipment, gas flow apparatus, and gas tanks, and therefore, some research groups have attempted to overcome the high-cost instrument problem and have proposed a more simple method for analysis of oxygen binding capacity in the laboratory $[28,31,32]$. 

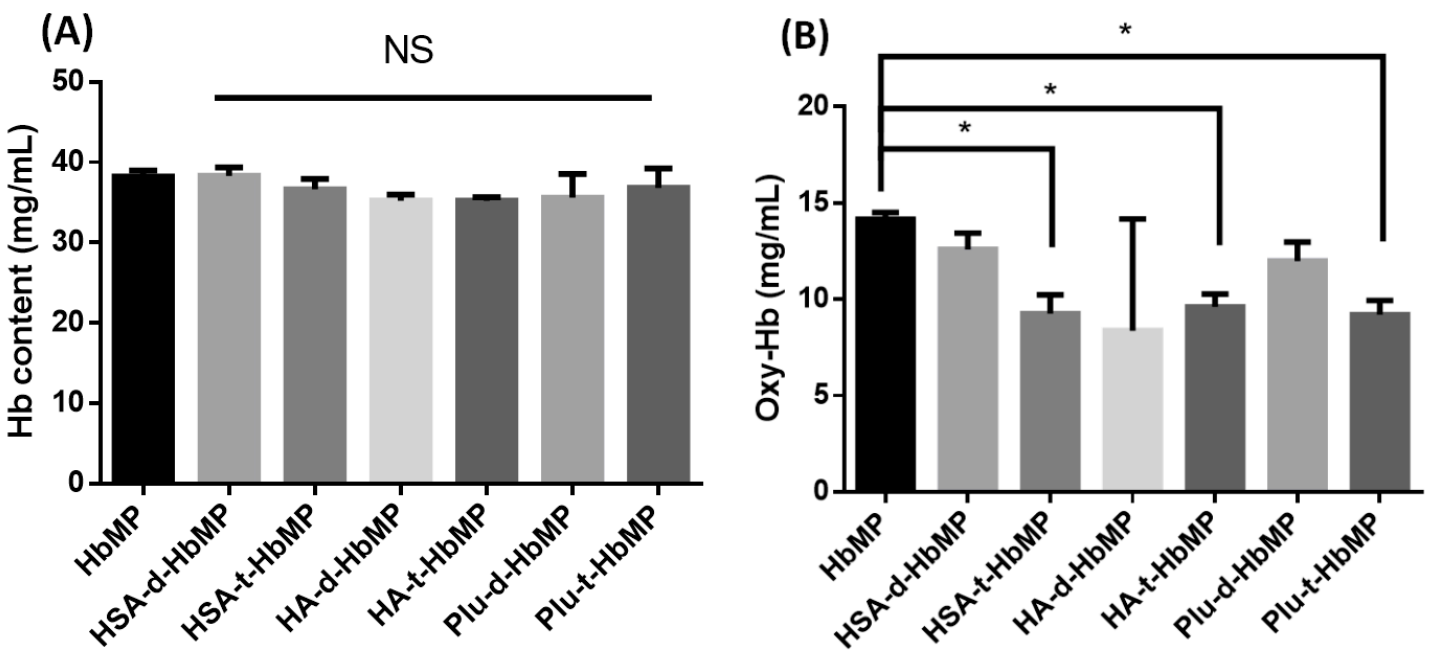

Figure 4. $\mathrm{The} \mathrm{Hb}(\mathbf{A})$ and oxygenated $\mathrm{Hb}(\mathrm{Oxy}-\mathrm{Hb})(\mathbf{B})$ content of surface-modified $\mathrm{HbMPs}$ as calculated for a suspension with an Hct of $20 \%$. Data are represented as mean \pm SD $(n=3)$. "NS" indicates not significant, whereas the asterisk indicates a significant difference when compared to $\mathrm{HbMPs}\left({ }^{*} p<0.05\right)$.

In this study, we applied the ferricyanide method to determine the concentration of $\mathrm{Oxy}-\mathrm{Hb}$ as a simple and inexpensive approach. The ferricyanide causes a conversion from oxyhemoglobin into met-hemoglobin (Met-Hb), and therefore a release of oxygen (as determined by the change in $p \mathrm{O}_{2}$ ) into the surrounding media, corresponding to the oxygen amount bound to hemoglobin in the particles. The oxygen binding capacity of the particles was determined from the content of $\mathrm{Oxy}-\mathrm{Hb}$ (Figure 4B). For all samples, the Oxy- $\mathrm{Hb}$ calculated for a suspension with a Hct of $20 \%$ was in a range between 9 to $12 \mathrm{mg} / \mathrm{mL}$. For the double precipitated HbMPs (HSA-d-HbMPs, HA-d-HbMPs and Plu-d-HbMP) the Oxy-Hb content showed a slight decreasing tendency, which was not statistically significant compared to the Oxy- $\mathrm{Hb}$ content of $\mathrm{HbMPs}$. In contrast, the $\mathrm{Oxy}-\mathrm{Hb}$ in the triple precipitated $\mathrm{HbMPs}$ showed a significant decrease by $33 \%, 32 \%$ and $35 \%$ for HSA-t-HbMPs, HA-t-HbMPs and Plu-t-HbMPs, respectively, when compared to HbMPs. This was most probably caused by the increased preparation time due to the additional coprecipitation and GA crosslinking steps during the surface modification, which can lead to partial oxidation of $\mathrm{Hb}$ to methemoglobin $(\mathrm{MetHb})$, where the iron in the heme group is in the ferric state [25,33].

\subsection{Binding of Anti-Hb Antibodies to Surface-Modified HbMPs}

The quality of the surface modification of the HbMPs was tested using indirect immunofluorescence staining with anti-Hb antibodies (Figure $5 \mathrm{~A}$ ). Unmodified $\mathrm{HbMPs}$ present $\mathrm{Hb}$ molecules on their surface that will be recognized by the rabbit anti-bovine $\mathrm{Hb}$ antibodies (primary antibodies). The fluorescently labeled secondary goat anti-rabbit antibodies subsequently recognize the primary antibodies on the surface of the HbMPs, and the fluorescence signal can be detected and analyzed by flow cytometry. 
(A)

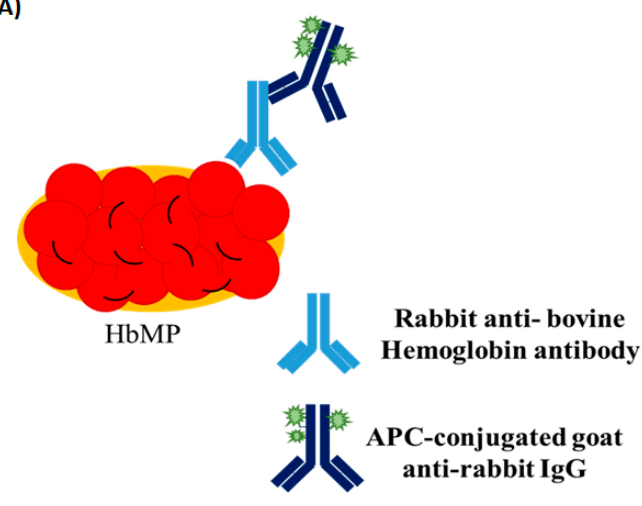

(B)

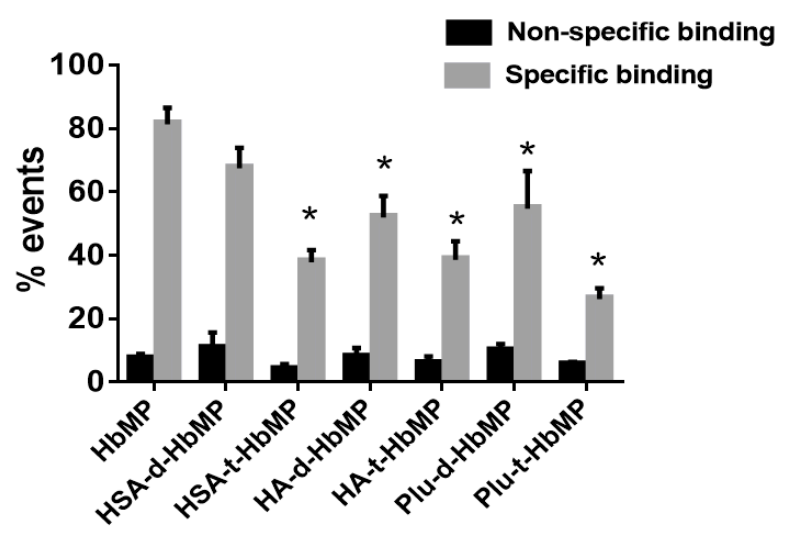

(C) HbMP

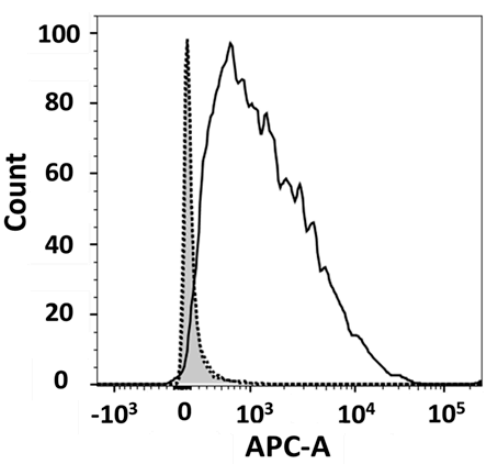

(D) HSA-d-HbMP

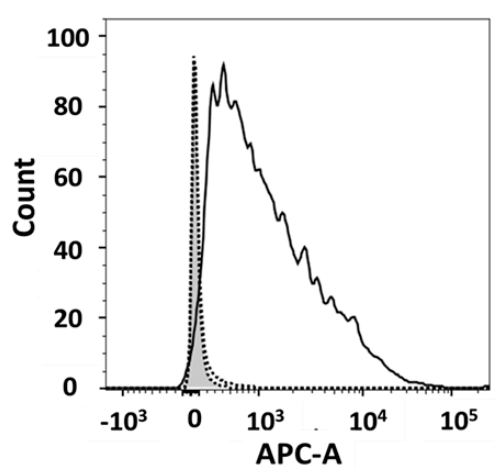

(E) HSA-t-HbMP

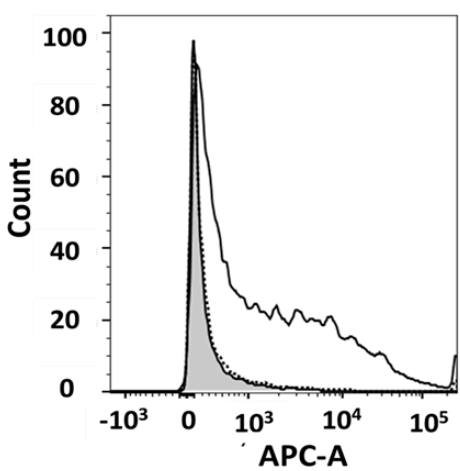

Figure 5. Immunofluorescence assay with rabbit anti-bovine-Hb antibodies. (A) Schematic drawing representing a model of indirect immunofluorescence staining with anti- $\mathrm{Hb}$ antibodies. (B) The \% positive events of non-specific and specific binding of anti-Hb antibodies on each surface-modified $\mathrm{HbMP}$. Specific binding was calculated by subtracting the non-specific binding from the total binding. Data represent the mean $\pm \mathrm{SD}$, and asterisks indicate the significance of differences when compared to HbMPs (* $p<0.05)$. Representative histograms show examples of flowcytometric analysis of anti- $\mathrm{Hb}$ antibodies binding on HbMPs (C), HSA-d-HbMPs (D) and HSA-t-HbMPs (E). Gray areas represent the histograms of particles that were not exposed to antibodies. Dash lines are histograms of particles with only secondary antibodies staining, as a non-specific binding. The solid lines are the histograms of particles with both primary and secondary antibody staining, as a total binding.

Figure 5B shows the percentage of non-specifically (only with secondary antibodies) and specifically stained particles with anti-Hb antibodies in each group (\% events). The $\%$ events positive for non-specific binding were low (5\%-10\%), with no statistically significant difference between the different samples. The $\%$ events positive for specific anti-Hb antibody binding were very high for the non-modified HbMPs $(\sim 80 \%)$ and for HSA-d-HbMPs $(\sim 70 \%)$, with no statistically significant difference. In the samples (HA-d-HbMP and Plu-d-HbMP) where double precipitation was performed, the $\%$ positive events of specific anti- $\mathrm{Hb}$ antibody binding was statistically significantly decreased when compared to $\mathrm{HbMPs}$ (by $36 \%$ and 33\%, respectively). Most importantly, the specific anti-Hb antibody binding in the samples modified by triple precipitation, HSA-t-HbMP, HA-t-HbMP and Plu-t-HbMP, was decreased by $54 \%, 56 \%$ and $67 \%$, respectively, when compared to the unmodified HbMPs. Additionally, the values of the main fluorescence intensity measured in all surface modified samples were lower than that measured for the HbMP sample (Supplementary Figure S2A). This is illustrated by representative histograms (Figure 5C-E) showing the distribution of the fluorescence intensity among the events of the HbMP, HSA-d-HbMP and HSA-t-HbMP samples. Therefore, the surface modifications by additional precipitations, especially by the triple precipitations, shielded the $\mathrm{Hb}$ molecules and inhibited their recognition by the anti-Hb antibodies. 


\subsection{Binding of IgG to Surface-Modified HbMPs}

The binding of IgG on the surface of particles leads to opsonization and recognition of the particles by the immunocompetent cells in the blood and tissues. Therefore, a high-quality surface modification should protect the particles from IgG adsorption. Here, we incubated our unmodified and modified HbMPs with biotinylated human IgG, which was subsequently detected by APC-labelled streptavidin (Figure 6A) and analyzed by flow cytometry.

(A)

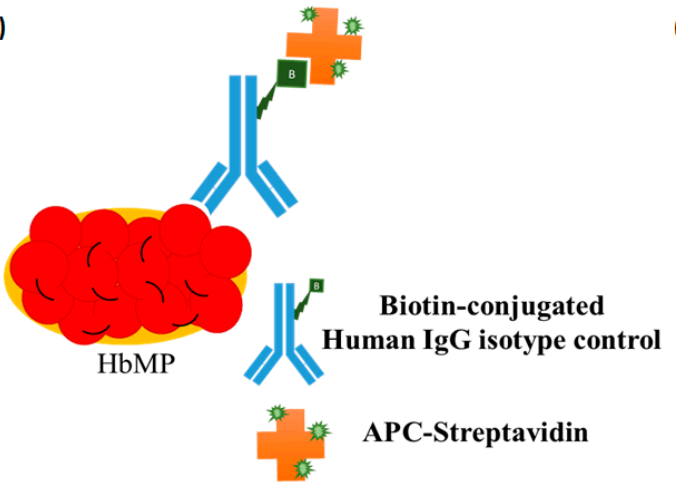

(B)

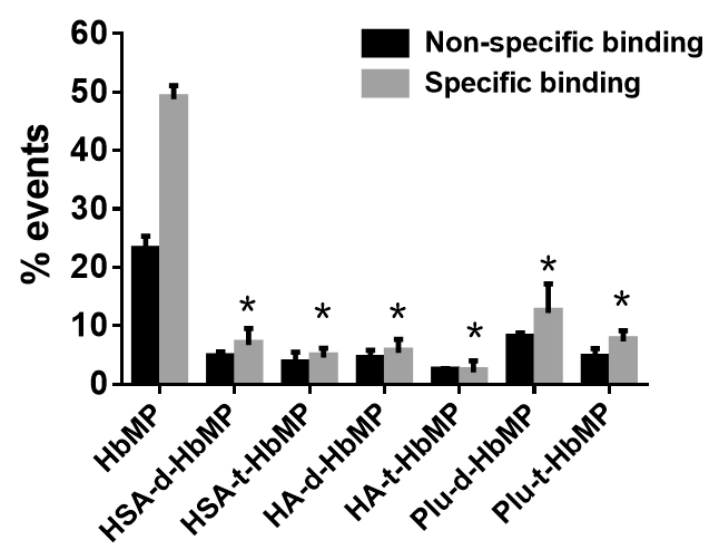

(C) HbMP

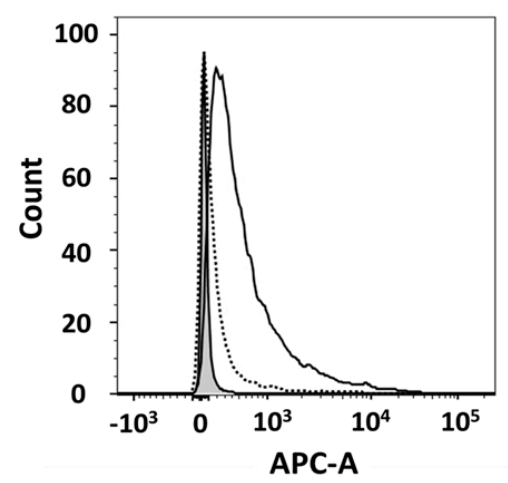

(D) HSA-d-HbMP

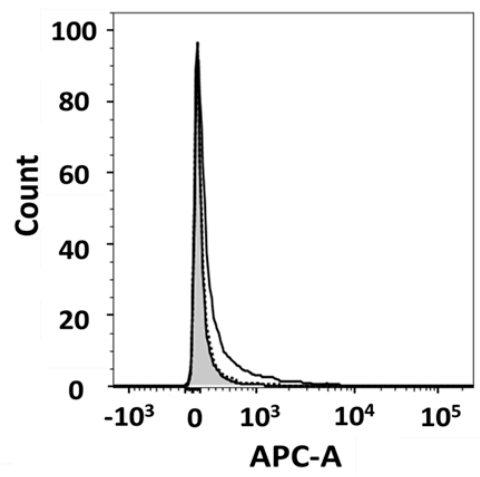

(E) HSA-t-HbMP

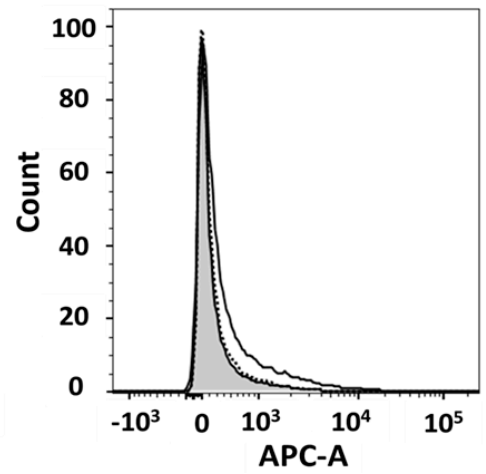

Figure 6. Immunofluorescence assay of the binding of IgG (biotinylated) to surface-modified HbMPs. (A) Schematic drawing representing the staining procedure with APC-Streptavidin. (B) The \% positive events of non-specific and specific binding of APC-Streptavidin on each surface-modified HbMP. Specific binding was calculated by subtracting the non-specific binding from the total binding. Data represent the mean $\pm \mathrm{SD}$, and asterisks indicate the significance of differences when compared to HbMPs (* $p<0.05$ ). Representative histograms show examples of flowcytometric analysis of the binding of APC-Streptavidin on HbMPs (C), HSA-d-HbMPs (D) and HSA-t-HbMPs (E). Gray areas represent the histograms of particles that were not exposed to antibodies. Dash lines are histograms of particles with only APC-Streptavidin staining, as a non-specific binding. The solid lines are the histograms of particles with both biotinylated IgG and APC-Streptavidin staining, as a total binding.

The results presented in Figure 6B,C show that IgG bound to the unmodified HbMPs (roughly $50 \%$ positive events of specific binding). In contrast, all surface modified samples showed statistically significant decreased events of specific binding by $80 \%-95 \%$. The values of the main fluorescence intensity measured in the surface modified samples were lower than that measured for the HbMP sample (Supplementary Figure S2B). This is illustrated by representative histograms (Figure 6C-E) showing the distribution of the fluorescence intensity among the events of the HbMP, HSA-d-HbMP, and HSA-t-HbMP samples. Therefore, the surface modification by additional precipitations successfully inhibited IgG binding to the particle surface. 


\subsection{Binding of Haptoglobin to Surface-Modified HbMPs}

$\mathrm{HP}$ is a glycoprotein that is mostly synthesized and secreted by liver cells, and plays a vital role with a high affinity to $\mathrm{Hb}$ in the circulation [34]. It is responsible for the rapid clearance of acellular $\mathrm{Hb}$ from the blood circulation, forming a complex with the $\beta$-chains of $\mathrm{Hb}$ which is then recognized by the Kupffer cells. Presumably, it can bind also to the $\mathrm{Hb}$ molecules on the surface of the HbMPs, which has to be avoided in order to achieve long circulation times of the HBOC.

We incubated our unmodified and modified HbMPs with human HP, which was subsequently detected by APC-labelled rabbit anti-human-HP antibodies (Figure 7A) and analyzed by flow cytometry. The results presented in Figure 7B,C show that HP bound to the unmodified HbMP (roughly $25 \%-30 \%$ positive events of specific binding). In contrast, all surface modified samples showed statistically significant decreased events of specific binding by $80 \%-95 \%$. The values of the main fluorescence intensity measured in the surface modified samples were lower than that measured for the HbMP sample (Supplementary Figure S2C). The representative histograms (Figure 7C-E) illustrate the distribution of the fluorescence intensity among the events of the HbMP, HSA-d-HbMP and HSA-t-HbMP samples. Therefore, the surface modification by additional precipitations successfully inhibited HP binding to the particle surface.

Our data demonstrated that a double/triple precipitation of HSA, HA and Plu on the surface of HbMPs can be successfully employed to shield the $\mathrm{Hb}$, resulting in a significant decrease of the specific binding of antibodies. In particular, the triple precipitated HbMPs showed the most promising results, reducing the binding of anti-Hb antibodies, IgG and HP, which can protect the HbMPs from endogeneous $\mathrm{Hb}$ removal mechanisms and rapid clearance from circulation.

The current development of blood substitutes, especially HBOCs, has amplified applications through investigation of the ability to transport oxygen, non-immunogenicity, extended half-life in circulation, and lack of toxicity. In this study, we modified the surface of HbMP by additional co-precipitation steps with three biopolymers (HSA, HA and Plu) and characterized their properties. Albumin and HA form complexes [35] and the amino groups of albumin are crosslinked by GA at the surface of HbMPs. That means HA will be immobilized at the surface, which was demonstrated by the zeta potential. In presence of HA, the zeta potential became more negative compared with that of only albumin. Pluronic also interacts with albumin [36], and the same mechanism should work as for HA, although only an indirect proof of the immobilization of Plu could be performed.

$\mathrm{HbMPs}$ were fabricated using the highly promising $\mathrm{CCD}$ technique based on $\mathrm{MnCO}_{3}$, which has been shown to be very simple and effective for entrapment of proteins into submicron particles $[21,25,30,37]$.

HBOCs may induce severe side effects and toxicity after administration, due to scavenging of vascular endothelial nitric oxide $(\mathrm{NO})$ and heme-mediated oxidative reactions [38,39]. Based on our previous reports of preclinical studies, HbMPs-700 fabricated by the CCD technique are a very promising approach, demonstrating a higher affinity to oxygen, avoidance of vasoconstriction, and non-mutagenicity [22,25].

Although HBOCs have shown a good biocompability and safety in pre-clinical studies, the circulatory clearance after administration is still a limitation, and it should be investigated for further clinical trials. In this study, we showed that surface modification of HbMPs could be a promising approach toward production of HBOCs with prolonged circulation. 

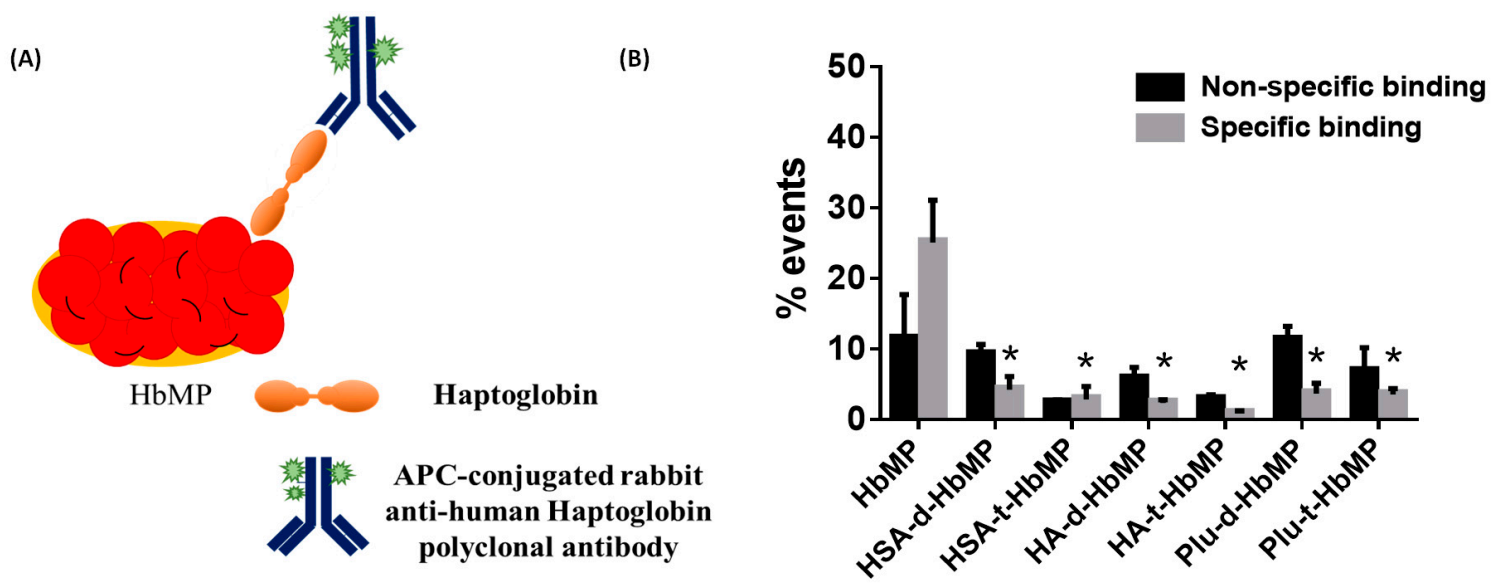

(C) HbMP

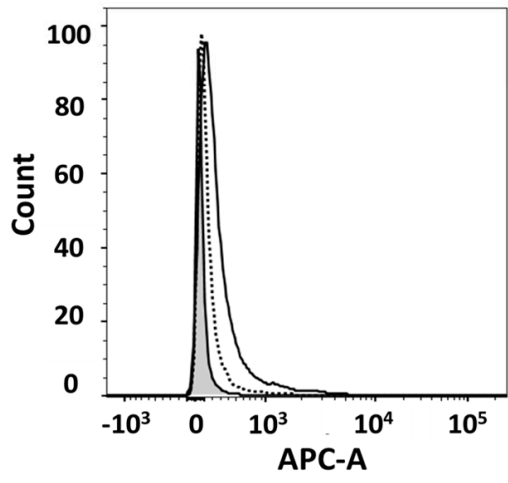

(D) HSA-d-HbMP

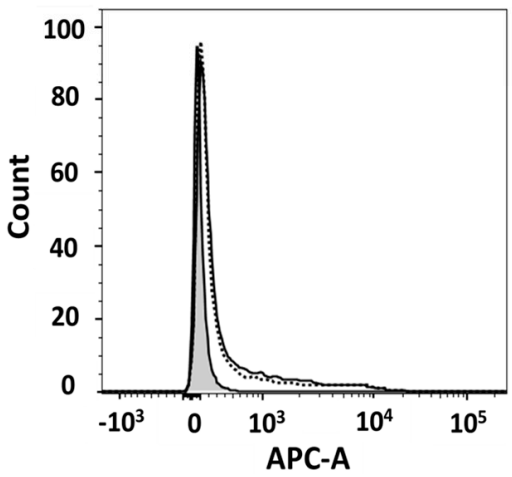

(E) HSA-t-HbMP

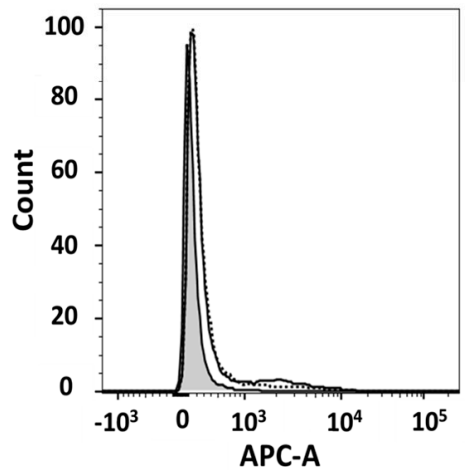

Figure 7. Immunofluorescence assay of haptoglobin (HP) binding to surface-modified HbMPs. (A) Schematic drawing representing the staining procedure. (B) The \% positive events of non-specific and specific binding of anti-HP antibodies on each surface-modified HbMP. Specific binding was calculated by subtracting the non-specific binding from the total binding. Data represent the mean \pm SD, and asterisks indicate the significance of differences when compared to HbMPs $\left({ }^{*} p<0.05\right)$. Representative histograms show example of flowcytometric analysis of anti-HP antibodies binding on HbMP (C), HSA-d-HbMP (D) and HSA-t-HbMP (E) samples. Gray areas represent the histogram of particles that were not exposed to antibodies. Dash lines are histograms of particles with only anti-HP antibody staining, as a non-specific binding. The solid lines are the histograms of particles with both HP and anti-HP antibodies staining, as a total binding.

\section{Conclusions}

Surface-modified HbMPs can be successfully prepared by modified CCD technique, including biopolymers, as shown here for HSA, HA and Plu, by means of double and triple co-precipitation. The obtained particles remained in the same size range as the unmodified HbMPs, with the typical peanut-like shape and more dense morphology.

The content of functional $\mathrm{Hb}$ of the surface-modified HbMPs was slightly decreased due to increased number of precipitations and additional GA crosslinking steps, leading to partial oxidation of $\mathrm{Hb}$ to $\mathrm{MetHb}$. This obstacle could be avoided by applying antioxidants during the preparation or by performing the fabrication under an oxygen free atmosphere.

The immunofluorescence assays revealed that our surface modification technique was efficient to reduce the specific binding of anti-Hb antibodies, IgG and HP. These results can be useful for predicting a longer half-life of HbMPs in circulation.

Supplementary Materials: The following are available online at http://www.mdpi.com/2079-6412/9/7/454/s1, Figure S1: Confocal laser scanning micrograph of HA-d-HbMPs (A,B), Plu-d-HbMPs (C,D), HA-t-HbMPs (E,F), 
and Plu-t-HbMPs $(\mathrm{G}, \mathrm{H})$ in fluorescence mode (left) and transmission mode (right) (Scale bar: $5 \mu \mathrm{m}$ ), Figure S2: The mean fluorescence intensity (MFI) of non-specific and specific binding of anti-Hb antibodies (A), IgG (B), and haptoglobin $(\mathrm{C})$ in each surface-modified HbMP is shown. Specific antibody binding values were calculated by subtracting the non-specific binding from total binding. Data represent the mean $\pm \mathrm{SD}$, and asterisks indicate the significance of differences when compared to HbMPs $\left({ }^{*} p<0.05\right)$.

Author Contributions: Experiments, Data Analysis and Writing, A.P. (Aussanai Prapan); Writing and Data Analysis, I.K.; Data Analysis, N.S., C.K., S.C., A.S. and Y.X.; Conceptualization, Design of Experiments, Performing Specific Experiments, Interpretation and Discussion of Results, Revision of Manuscript, R.G.; Evaluation and Discussion of Results, A.P. (Axel Pruß); Conceptualization, Advised all Research, Design of Experiments, Evaluation and Discussion of Results, Revision of Manuscript, H.B.

Funding: A.P. (Aussanai Prapan) hold an academic development scholarship from Naresuan University, N.S. and C.K. from University of Phayao, S.C. from Payap University. This research received no external funding.

Acknowledgments: We are grateful to Petra Schrade, Core Facility of Electron Microscopy, Institute of Anatomy for the TEM images. We thank Chutamas Thepmalee and Patcharin Thammasit for suggestion on the result of immunofluorescence staining. We acknowledge support from the German Research Foundation (DFG) and the Open Access Publication Funds of Charité-Universitätsmedizin Berlin

Conflicts of Interest: The authors declare no conflict of interest.

\section{References}

1. Sharma, S.; Sharma, P.; Tyler, L.N. Transfusion of blood and blood products: Indications and complications. Am. Fam. Physician 2011, 83, 719-724. [CrossRef] [PubMed]

2. Rawn, J. The silent risks of blood transfusion. Curr. Opin. Anaesthesiol. 2008, 21, 664-668. [CrossRef] [PubMed]

3. Moradi, S.; Jahanian-Najafabadi, A.; Roudkenar, M.H. Artificial Blood Substitutes: First steps on the long route to clinical utility. Clin. Med. Insights Blood Disord. 2016, 9, 33-41. [CrossRef] [PubMed]

4. Seifried, E.; Mueller, M.M. The present and future of transfusion medicine. Blood Transfus. 2011, 9, 371-376. [CrossRef] [PubMed]

5. Winslow, R.M. Current status of oxygen carriers. Vox Sang. 2006, 91, 102-110. [CrossRef]

6. Keipert, P.E. Hemoglobin-Based Oxygen Carrier (HBOC) Development in Trauma: Previous Regulatory Challenges, Lessons Learned, and a Path Forward; Advances in Experimental Medicine and Biology Series 977; Springer: Cham, Switzerland, 2017; pp. 343-350. [CrossRef]

7. Chen, J.-Y.; Scerbo, M.; Kramer, G. A review of blood substitutes: Examining the history, clinical trial results, and ethics of hemoglobin-based oxygen carriers. Clinics 2009, 64, 803-814. [CrossRef]

8. Alayash, A.I. Blood substitutes: Why haven't we been more successful? Trends Biotechnol. 2014, 32, 177-185. [CrossRef]

9. Jia, Y.; Duan, L.; Li, J. Hemoglobin-based nanoarchitectonic assemblies as oxygen carriers. Adv. Mater. 2016, 28, 1312-1318. [CrossRef]

10. Estep, T.N. Pharmacokinetics and mechanisms of plasma removal of hemoglobin-based oxygen carriers. Artif. Cells Nanomed. Biotechnol. 2015, 43, 203-215. [CrossRef]

11. Shih, A.W.Y.; Mcfarlane, A.; Verhovsek, M. Haptoglobin testing in hemolysis: Measurement and interpretation. Am. J. Hematol. 2014, 89, 443-447. [CrossRef]

12. Schaer, D.J.; Vinchi, F.; Ingoglia, G.; Tolosano, E.; Buehler, P.W. Haptoglobin, hemopexin, and related defense pathways—basic science, clinical perspectives, and drug development. Front. Physiol. 2014, 5, 415. [CrossRef] [PubMed]

13. Salmaso, S.; Caliceti, P. Stealth properties to improve therapeutic efficacy of drug nanocarriers. J. Drug Deliv. 2013, 2013, 374252. [CrossRef] [PubMed]

14. Amoozgar, Z.; Yeo, Y. Recent advances in stealth coating of nanoparticle drug delivery systems. Wiley Interdiscip. Rev. Nanomed. Nanobiotechnol. 2012, 4, 219-233. [CrossRef] [PubMed]

15. Chong, B.F.; Blank, L.M.; Mclaughlin, R.; Nielsen, L.K. Microbial hyaluronic acid production. Appl. Microbiol. Biotechnol. 2005, 66, 341-351. [CrossRef] [PubMed]

16. Szarpak, A.; Cui, D.; Dubreuil, F.; De Geest, B.G.; De Cock, L.J.; Picart, C.; Auzély-Velty, R. Designing hyaluronic acid-based layer-by-layer capsules as a carrier for intracellular drug delivery. Biomacromolecules 2010, 11, 713-720. [CrossRef] [PubMed] 
17. Gyulai, G.; Magyar, A.; Rohonczy, J.; Orosz, J.; Yamasaki, M.; Bősze, S.; Kiss, E. Preparation and characterization of cationic pluronic for surface modification and functionalization of polymeric drug delivery nanoparticles. Express Polym. Lett. 2016, 10, 216-226. [CrossRef]

18. Nitta, S.K.; Numata, K. Biopolymer-based nanoparticles for drug/gene delivery and tissue engineering. Int. J. Mol. Sci. 2013, 14, 1629-1654. [CrossRef] [PubMed]

19. Liu, F.; Mu, J.; Xing, B. Recent advances on the development of pharmacotherapeutic agents on the basis of human serum albumin. Curr. Pharm. Des. 2015, 21, 1866-1888. [CrossRef]

20. Esfandyari-manesh, M.; Mostafavi, S.H.; Majidi, R.F.; Koopaei, M.N.; Ravari, N.S.; Amini, M.; Darvishi, B.; Ostad, S.N.; Atyabi, F.; Dinarvand, R. Improved anticancer delivery of paclitaxel by albumin surface modification of PLGA nanoparticles. DARU J. Pharm. Sci. 2015, 23, 28. [CrossRef]

21. Bäumler, H.; Xiong, Y.; Liu, Z.Z.; Patzak, A.; Georgieva, R. Novel hemoglobin particles-promising new-generation hemoglobin-based oxygen carriers. Artif. Organs 2014, 38, 708-714. [CrossRef]

22. Kao, I.; Xiong, Y.; Steffen, A.; Smuda, K.; Zhao, L.; Georgieva, R.; Pruss, A.; Bäumler, H. Preclinical in vitro safety investigations of submicron sized hemoglobin based oxygen carrier HbMP-700. Artif. Organs 2018, 42, 549-559. [CrossRef] [PubMed]

23. Haney, C.R.; Buehler, P.W.; Gulati, A. Purification and chemical modifications of hemoglobin in developing hemoglobin based oxygen carriers. Adv. Drug Deliv. Rev. 2000, 40, 153-169. [CrossRef]

24. Bäumler, H.; Georgieva, R. Coupled enzyme reactions in multicompartment microparticles. Biomacromolecules 2010, 11, 1480-1487. [CrossRef] [PubMed]

25. Xiong, Y.; Steffen, A.; Andreas, K.; Müller, S.; Sternberg, N.; Georgieva, R.; Bäumler, H. Hemoglobin-based oxygen carrier microparticles: Synthesis, properties, and in vitro and in vivo investigations. Biomacromolecules 2012, 13, 3292-3300. [CrossRef] [PubMed]

26. Kloypan, C.; Prapan, A.; Suwannasom, N.; Chaiwaree, S.; Kaewprayoon, W.; Steffen, A.; Xiong, Y.; Baisaeng, N.; Georgieva, R.; Bäumler, H. Improved oxygen storage capacity of haemoglobin submicron particles by one-pot formulation. Artif. Cells Nanomed. Biotechnol. 2018, 46, S964-S972. [CrossRef] [PubMed]

27. Anchinmane, V.T.; Sankhe, S.V. Evaluation of hemoglobin estimation with non-cyanide alkaline haematin D-575 method. Int. J. Res. Med. Sci. 2016, 44, 4297-4299. [CrossRef]

28. Haldane, J. The ferricyanide method of determining the oxygen capacity of blood. J Physiol. 1900, 25, $295-302$. [CrossRef]

29. Baidukova, O.; Wang, Q.; Chaiwaree, S.; Freyer, D.; Prapan, A.; Georgieva, R.; Zhao, L.; Baumler, H. Antioxidative protection of haemoglobin microparticles (HbMPs) by polydopamine. Artif. Cells Nanomed. Biotechnol. 2018, 46, S693-S701. [CrossRef]

30. Xiong, Y.; Georgieva, R.; Steffen, A.; Smuda, K.; Bäumler, H. Structure and properties of hybrid biopolymer particles fabricated by co-precipitation cross-linking dissolution procedure. J. Colloid Interface Sci. 2018, 514, 156-164. [CrossRef]

31. Cook, S.F. The action of potassium cyanide on certain respiratory pigments. J. Gen. Physiol. 1928, 11, 339-348. [CrossRef]

32. Zhang, X.; Liu, C.; Yuan, Y.; Shan, X.; Sheng, Y.; Xu, F. A noninvasive method for measuring the oxygen binding-releasing capacity of hemoglobin-loaded polymeric nanoparticles as oxygen carrier. J. Mater. Sci. Mater. Med. 2009, 20, 1025-1030. [CrossRef] [PubMed]

33. Wu, M.; Feng, K.; Li, Q.; Ma, H.; Zhu, H.; Xie, Y.; Yan, G.; Chen, C.; Yan, K. Glutaraldehyde-polymerized hemoglobin and tempol (PolyHb-Tempol) has superoxide dismutase activity that can attenuate oxidative stress on endothelial cells induced by superoxide anion. Artif. Cells Nanomed. Biotechnol. 2018, 46, 47-55. [CrossRef] [PubMed]

34. Jia, Y.; Wood, F.; Buehler, P.W.; Alayash, A.I. Haptoglobin preferentially binds $\beta$ but not $\alpha$ subunits cross-linked hemoglobin tetramers with minimal effects on ligand and redox reactions. PLoS ONE 2013,8, e59841. [CrossRef] [PubMed]

35. Pigman, W.; Gramling, E.; Holley, H.L. Interactions of hyaluronic acid with serum albumin. Biochim. Biophys. Acta 1961, 46, 100-107. [CrossRef]

36. Neacsu, M.V.; Matei, I.; Micutz, M.; Staicu, T.; Precupas, A.; Popa, V.T.; Salifoglou, A.; Ionita, G. Interaction between albumin and pluronic F127 block copolymer revealed by global and local physicochemical profiling. J. Phys. Chem. B 2016, 120, 4258-4267. [CrossRef] [PubMed] 
37. Xiong, Y.; Liu, Z.Z.; Georgieva, R.; Smuda, K.; Steffen, A.; Sendeski, M.; Voigt, A.; Patzak, A.; Bäumler, H. Nonvasoconstrictive hemoglobin particles as oxygen carriers. ACS Nano 2013, 7, 7454-7461. [CrossRef] [PubMed]

38. Alayash, A.I. Mechanisms of toxicity and modulation of hemoglobin-based oxygen carriers (HBOCs). Shock 2017, 3, 1-16. [CrossRef]

39. Toma, V.A.; Farca, A.D.; Roman, I.; Sevastre, B.; Hathazi, D.; Scurtu, F.; Damian, G.; Silaghi-Dumitrescu, R. Comparative in vivo effects of hemoglobin- based oxygen carriers ( HBOC ) with varying prooxidant and physiological reactivity. PLoS ONE 2016, 11, e0153909. [CrossRef]

(C) 2019 by the authors. Licensee MDPI, Basel, Switzerland. This article is an open access article distributed under the terms and conditions of the Creative Commons Attribution (CC BY) license (http://creativecommons.org/licenses/by/4.0/). 